\title{
REPRESENTATION THEORY OF SYMMETRIC GROUPS AND THEIR DOUBLE COVERS
}

\author{
JONATHAN BRUNDAN AND ALEXANDER KLESHCHEV
}

\section{INTRODUCTION}

In this article we will give an overview of the new Lie theoretic approach to the $p$-modular representation theory of the symmetric groups and their double covers that has emerged in the last few years. There are in fact two parallel theories here: one for the symmetric groups $S_{n}$ involving the affine Kac-Moody algebra of type $A_{p-1}^{(1)}$, and one for their double covers $\widehat{S}_{n}$ involving the twisted algebra of type $A_{p-1}^{(2)}$. In the case of $S_{n}$ itself, the theory has been developed especially by Kleshchev [19], Lascoux-Leclerc-Thibon [21], Ariki [1] and Grojnowski [9], while the double covers are treated for the first time in [4] along the lines of [9], after the important progress made over $\mathbb{C}$ by Sergeev $[35,36]$ and Nazarov $[30,31]$.

One of the most striking results at the heart of both of the theories is the explicit description of the modular branching graphs in terms of Kashiwara's crystal graph for the basic module of the corresponding affine Lie algebra. Note that the results described are just a part of a larger picture: there are analogous results for the cyclotomic and affine Hecke algebras, and their twisted analogues, the cyclotomic and affine Hecke-Clifford superalgebras. However we will try here to bring out only those parts of the theory that apply to the symmetric group, since that is the most applicable to finite group theory.

\section{The SYMMETRIC GROUP}

In this section, we describe the representation theory of the symmetric group $S_{n}$ over a field $F$ of arbitrary characteristic $p$.

2.1. Formal characters. For $k=1, \ldots, n$, we define the Jucys-Murphy element

$$
x_{k}:=\sum_{i=1}^{k-1}(i k) \in F S_{n},
$$

see $[15,28]$. It is straightforward to show that the elements $x_{1}, x_{2}, \ldots, x_{n}$ commute with one another. Moreover, we have by [15] or [29, 1.9]:

Theorem 2.1. The center of the group algebra $F S_{n}$ is precisely the set of all symmetric polynomials in the elements $x_{1}, x_{2}, \ldots, x_{n}$. 
Now let $M$ be an $F S_{n}$-module. Let $I=\mathbb{Z} / p \mathbb{Z}$ identified with the prime subfield of $F$. For $\underline{i}=\left(i_{1}, \ldots, i_{n}\right) \in I^{n}$, define

$$
M[\underline{i}]:=\left\{v \in M \mid\left(x_{r}-i_{r}\right)^{N} v=0 \text { for } N \gg 0 \text { and each } r=1, \ldots, n\right\} .
$$

Thus, $M[\underline{i}]$ is the simultaneous generalized eigenspace for the commuting operators $x_{1}, \ldots, x_{n}$ corresponding to the eigenvalues $i_{1}, \ldots, i_{n}$ respectively.

Lemma 2.2. Any $F S_{n}$-module $M$ decomposes as $M=\bigoplus_{\underline{i} \in I^{n}} M[\underline{i}]$.

Proof. It suffices to show that all eigenvalues of $x_{r}$ on $M$ lie in $I$, for each $r=1, \ldots, n$. This is obvious if $r=1$ (as $x_{1}=0$ ). Now assume that all eigenvalues of $x_{r}$ on $M$ lie in $I$, and consider $x_{r+1}$. Let $v \in M$ be a simultaneous eigenvector for the commuting operators $x_{r}$ and $x_{r+1}$. Consider the subspace $N$ spanned by $v$ and $s_{r} v$.

Suppose that $N$ is two dimensional. Then the matrix for the action of $x_{r}$ on $N$ with respect to the basis $\left\{v, s_{r} v\right\}$ is $\left(\begin{array}{cc}i & c \\ 0 & j\end{array}\right)$ for some $i, j \in I$ and $c \in F$ (by assumption on the eigenvalues of $x_{r}$ ). Hence, the matrix for the action of $x_{r+1}=s_{r} x_{r} s_{r}+s_{r}$ on $N$ is $\left(\begin{array}{ll}j & 1 \\ c+1 & i\end{array}\right)$. Since $v$ was an eigenvector for $x_{r+1}$, we see that $c=-1$, hence $v$ has eigenvalue $j$ for $x_{r+1}$ as required.

Finally suppose that $N$ is one dimensional. Then, $s_{r} v= \pm v$. Hence, if $x_{r} v=i v$ for $i \in I$, then $x_{r+1} v=\left(s_{r} x_{r} s_{r}+s_{r}\right) v=(i \pm 1) v$. Since $i \pm 1 \in I$, we are done.

We define the formal character $\operatorname{ch} M$ of a finite dimensional $F S_{n}$-module $M$ to be

$$
\operatorname{ch} M:=\sum_{\underline{i} \in I^{n}} \operatorname{dim}(M[\underline{i}]) e^{\underline{i}},
$$

an element of the free $\mathbb{Z}$-module on basis $\left\{e^{\underline{i}} \mid \underline{i} \in I^{n}\right\}$. This is a useful notion, since ch is clearly additive on short exact sequences and we have the following important result proved in $[38, \S 5.5]$ :

Theorem 2.3. The formal characters of the inequivalent irreducible $F S_{n^{-}}$ modules are linearly independent.

Given $\underline{i}=\left(i_{1}, \ldots, i_{n}\right) \in I^{n}$, define its weight wt $(\underline{i})$ to be the tuple $\gamma=$ $\left(\gamma_{i}\right)_{i \in I}$ where $\gamma_{j}$ counts the number of $i_{r}(r=1, \ldots, n)$ that equal $j$. Thus, $\gamma$ is an element of the set $\Gamma_{n}$ of $I$-tuples of non-negative integers summing to $n$. Clearly $\underline{i}, \underline{j} \in I^{n}$ lie in the same $S_{n}$-orbit (under the obvious action by place permutation) if and only if $\operatorname{wt}(\underline{i})=\operatorname{wt}(\dot{j})$, hence $\Gamma_{n}$ parametrizes the

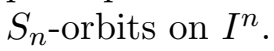

For $\gamma \in \Gamma_{n}$ and an $F S_{n}$-module $M$, we let

$$
M[\gamma]:=\sum_{\underline{i} \in I^{n}} \sum_{\text {with } \operatorname{wt}(\underline{i})=\gamma} M[\underline{i}] .
$$


Unlike the $M[\underline{i}]$, the subspaces $M[\gamma]$ are actually $F S_{n}$-submodules of $M$. Indeed, as an elementary consequence of Theorem 2.1 and Lemma 2.2, we have:

Lemma 2.4. The decomposition $M=\bigoplus_{\gamma \in \Gamma_{n}} M[\gamma]$ is precisely the decomposition of $M$ into blocks as an $F S_{n}$-module.

We will say that an $F S_{n}$-module $M$ belongs to the block $\gamma$ if $M=M[\gamma]$.

2.2. Induction and restriction operators. Now that we have the notion of formal character, we can introduce the $i$-restriction and $i$-induction operators $e_{i}$ and $f_{i}$. Suppose that $\gamma \in \Gamma_{n}$. Let $\gamma+i \in \Gamma_{n+1}$ be the tuple $\left(\delta_{i}\right)_{i \in I}$ with $\delta_{j}=\gamma_{j}$ for $j \neq i$ and $\delta_{i}=\gamma_{i}+1$. Similarly, assuming this time that $\gamma_{i}>0$, let $\gamma-i \in \Gamma_{n-1}$ be the tuple $\left(\delta_{i}\right)_{i \in I}$ with $\delta_{j}=\gamma_{j}$ for $j \neq i$ and $\delta_{i}=\gamma_{i}-1$.

If $M$ is an $F S_{n}$-module belonging to the block $\gamma \in \Gamma_{n}$, define

$$
\begin{aligned}
e_{i} M & \left.:=\left(\operatorname{res}_{S_{n-1}}^{S_{n}} M\right)[\gamma-i] \quad \text { (interpreted as } 0 \text { in case } \gamma_{i}=0\right), \\
f_{i} M & :=\left(\operatorname{ind}_{S_{n}}^{S_{n+1}} M\right)[\gamma+i] .
\end{aligned}
$$

Extending additively to arbitrary $F S_{n}$-modules $M$ using Lemma 2.4 and making the obvious definition on morphisms, we obtain exact functors

$$
e_{i}: F S_{n}-\bmod \rightarrow F S_{n-1} \text {-mod } \quad \text { and } \quad f_{i}: F S_{n}-\bmod \rightarrow F S_{n+1} \text {-mod } .
$$

The definition implies:

Lemma 2.5. For an $F S_{n}$-module $M$ we have

$$
\operatorname{res}_{S_{n-1}}^{S_{n}} M \cong \bigoplus_{i \in I} e_{i} M, \quad \operatorname{ind}_{S_{n}}^{S_{n+1}} M \cong \bigoplus_{i \in I} f_{i} M .
$$

Note that $e_{i} M$ can be described alternatively as the generalized eigenspace of $x_{n}$ acting on $M$ corresponding to the eigenvalue $i$. This means that the effect of $e_{i}$ on characters is easy to describe:

$$
\text { if } \operatorname{ch} M=\sum_{\underline{i} \in I^{n}} a_{\underline{i}} e^{\underline{i}} \quad \text { then } \quad \operatorname{ch}\left(e_{i} M\right)=\sum_{\underline{i} \in I^{n-1}} a_{\left(i_{1}, \ldots, i_{n-1}, i\right)} e^{\underline{i}} .
$$

Let us also mention that there are higher divided power functors $e_{i}^{(r)}, f_{i}^{(r)}$ for each $r \geq 1$. To define them, start with an $F S_{n}$-module $M$ belonging to the block $\gamma$. Let $\gamma+i^{r}=\gamma+i+i+\cdots+i$ ( $r$ times), and define $\gamma-i^{r}$ similarly (assuming $\left.\gamma_{i} \geq r\right)$. View $M$ instead as an $F\left(S_{n} \times S_{r}\right)$-module by letting $S_{r}$ act trivially. Embedding $S_{n} \times S_{r}$ into $S_{n+r}$ in the obvious way, we then define

$$
f_{i}^{(r)} M:=\left(\operatorname{ind}_{S_{n} \times S_{r}}^{S_{n+r}} M\right)\left[\gamma+i^{r}\right] .
$$

Extending additively, we obtain the functor $f_{i}^{(r)}: F S_{n}$-mod $\rightarrow F S_{n+r}$-mod . This exact functor has a two-sided adjoint $e_{i}^{(r)}: F S_{n+r}$-mod $\rightarrow F S_{n}$-mod. It is defined on a module $M$ belonging to block $\gamma$ by

$$
\left.e_{i}^{(r)} M:=\left(M^{S_{r}}\right)\left[\gamma-i^{r}\right] \quad \text { (interpreted as zero if } \gamma_{i}<r\right),
$$


where $M^{S_{r}}$ denotes the space of fixed points for the subgroup $S_{r}<S_{n+r}$ that permutes $n+1, \ldots, n+r$, viewed as a module over the subgroup $S_{n}<S_{n+r}$ that permutes $1, \ldots, n$. The following lemma relates the divided power functors $e_{i}^{(r)}$ and $f_{i}^{(r)}$ to the original functors $e_{i}, f_{i}$ :

Lemma 2.6. For an $F S_{n}$-module $M$ we have

$$
e_{i}^{r} M \cong\left(e_{i}^{(r)} M\right)^{\oplus r !}, \quad f_{i}^{r} M \cong\left(f_{i}^{(r)} M\right)^{\oplus r !} .
$$

The functors $e_{i}^{(r)}$ and $f_{i}^{(r)}$ have been defined in an entirely different way by Grojnowski $[9, \S 8.1]$, which is the key to proving their properties including Lemma 2.6.

2.3. The affine Kac-Moody algebra. Let $R_{n}$ denote the character ring of $F S_{n}$, i.e. the free $\mathbb{Z}$-module spanned by the formal characters of the irreducible $F S_{n}$-modules. In view of Theorem 2.3, the map ch induces an isomorphism between $R_{n}$ and the Grothendieck group of the category of all finite dimensional $F S_{n}$-modules. Similarly, let $R_{n}^{*}$ denote the $\mathbb{Z}$-submodule of $R_{n}$ spanned by the formal characters of the projective indecomposable $F S_{n}$-modules. This time, the map ch induces an isomorphism between $R_{n}^{*}$ and the Grothendieck group of the category of all finite dimensional projective $F S_{n}$-modules.

Let

$$
R=\bigoplus_{n \geq 0} R_{n}, \quad R^{*}=\bigoplus_{n \geq 0} R_{n}^{*} \subseteq R .
$$

The exact functors $e_{i}$ and $f_{i}$ induce $\mathbb{Z}$-linear operators on $R$. Since induction and restriction send projective modules to projective modules, Lemma 2.5 implies that $e_{i}$ and $f_{i}$ do too. Hence, $R^{*} \subseteq R$ is invariant under the action of $e_{i}$ and $f_{i}$.

Extending scalars we get $\mathbb{C}$-linear operators $e_{i}$ and $f_{i}$ on $R_{\mathbb{C}}:=\mathbb{C} \otimes_{\mathbb{Z}} R=$ $\mathbb{C} \otimes_{\mathbb{Z}} R^{*}$. There is also a non-degenerate symmetric bilinear form on $R_{\mathbb{C}}$, the usual Cartan pairing, with respect to which the characters of the projective indecomposables and the irreducibles form a pair of dual bases.

Theorem 2.7. The operators $e_{i}$ and $f_{i}(i \in I)$ on $R_{\mathbb{C}}$ satisfy the defining relations of the Chevalley generators of the affine Kac-Moody Lie algebra $\mathfrak{g}$ of type $A_{p-1}^{(1)}\left(\right.$ resp. $A_{\infty}$ in case $\left.p=0\right)$, see [16]. Moreover, viewing $R_{\mathbb{C}}$ as a $\mathfrak{g}$-module in this way,

(i) $R_{\mathbb{C}}$ is isomorphic to the basic representation $V\left(\Lambda_{0}\right)$ of $\mathfrak{g}$, generated by the highest weight vector $e^{0}$ (the character of the irreducible $F S_{0^{-}}$ module);

(ii) the decomposition of $R_{\mathbb{C}}$ into blocks coincides with its weight space decomposition with respect to the standard Cartan subalgebra of $\mathfrak{g}$;

(iii) the Cartan pairing on $R_{\mathbb{C}}$ coincides with the Shapovalov form satisfying $\left(e^{0}, e^{0}\right)=1$

(iv) the lattice $R^{*} \subset R_{\mathbb{C}}$ is the $\mathbb{Z}$-submodule of $R_{\mathbb{C}}$ generated by $e^{0}$ under the action of the operators $f_{i}^{(r)}=f_{i}^{r} / r !(i \in I, r \geq 0)$; 
(v) the lattice $R \subset R_{\mathbb{C}}$ is the dual lattice to $R^{*}$ under the Shapovalov form.

This was essentially proved by Lascoux-Leclerc-Thibon [21] and Ariki [1] (for a somewhat different situation), and another approach has been given more recently by Grojnowski $[9,14.2],[10]$.

2.4. The crystal graph. In view of Theorem 2.7 , we can identify $R_{\mathbb{C}}$ with the basic representation of the affine Kac-Moody algebra $\mathfrak{g}=A_{p-1}^{(1)}$. Associated to this highest weight module, Kashiwara has defined a purely combinatorial object known as a crystal, see e.g. [18] for a survey of this amazing theory. We now review the explicit description of this particular crystal, due originally to Misra and Miwa [26]. This contains all the combinatorial notions we need to complete our exposition of the representation theory.

Let $\lambda=\left(\lambda_{1} \geq \lambda_{2} \geq\right)$ be a partition. We identify $\lambda$ with its Young diagram

$$
\lambda=\left\{(r, s) \in \mathbb{Z}_{>0} \times \mathbb{Z}_{>0} \mid s \leq \lambda_{r}\right\} .
$$

Elements $(r, s) \in \mathbb{Z}_{>0} \times \mathbb{Z}_{>0}$ are called nodes. We label each node $A=(r, s)$ of $\lambda$ with its residue res $A \in I$ defined so that res $A \equiv(s-r)(\bmod p)$, see Example 2.8 below.

Let $i \in I$ be some fixed residue. A node $A \in \lambda$ is called $i$-removable (for $\lambda)$ if

(R0) res $A=i$ and $\lambda-\{A\}$ is the diagram of a partition.

Similarly, a node $B \notin \lambda$ is called $i$-addable (for $\lambda$ ) if

(A0) res $B=i$ and $\lambda \cup\{B\}$ is the diagram of a partition.

Now label all $i$-addable nodes of the diagram $\lambda$ by + and all $i$-removable nodes by - . The $i$-signature of $\lambda$ is the sequence of pluses and minuses obtained by going along the rim of the Young diagram from bottom left to top right and reading off all the signs. The reduced $i$-signature of $\lambda$ is obtained from the $i$-signature by successively erasing all neighbouring pairs of the form -+ .

Example 2.8. Let $p=3$ and $\lambda=(11,10,9,9,5,1)$. The residues are as follows:

\begin{tabular}{|c|c|c|c|c|c|c|c|c|c|c|}
\hline 0 & 1 & 2 & 0 & 1 & 2 & 0 & 1 & 2 & & 1 \\
\hline 2 & 0 & 1 & 2 & 0 & 1 & 2 & 0 & 1 & 2 & \\
\hline 1 & 2 & 0 & 1 & 2 & 0 & 1 & 2 & 0 & & \\
\hline 0 & 1 & 2 & 0 & 1 & 2 & 0 & 1 & 2 & & \\
\hline 2 & 0 & 1 & 2 & 0 & & & & & & \\
\hline 1 & & & & & & & & & & \\
\hline
\end{tabular}


The 2-addable and 2-removable nodes are as labelled in the diagram:

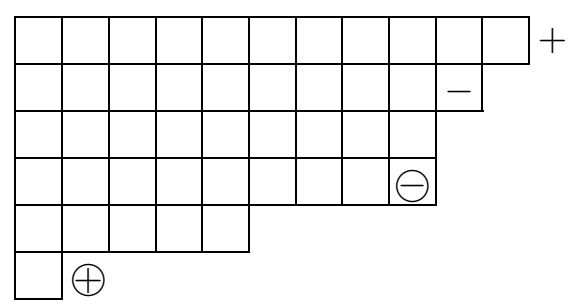

Hence, the 2-signature of $\lambda$ is,,,+--+ and the reduced 2 -signature is,+(the nodes corresponding to the reduced 2-signature have been circled in the above diagram).

Note the reduced $i$-signature always looks like a sequence of + 's followed by -'s. Nodes corresponding to a - in the reduced $i$-signature are called $i$-normal, nodes corresponding to a + are called $i$-conormal. The leftmost $i$-normal node (corresponding to the leftmost - in the reduced $i$-signature) is called $i$-good, and the rightmost $i$-conormal node (corresponding to the rightmost + in the reduced $i$-signature) is called $i$-cogood.

We recall finally that a partition $\lambda$ is called $p$-regular if it does not have $p$ non-zero equal parts. It is important to note that if $\lambda$ is $p$-regular and $A$ is an $i$-good node, then $\lambda-\{A\}$ is also $p$-regular. Similarly if $B$ is an $i$-cogood node, then $\lambda \cup\{B\}$ is $p$-regular.

By [26], the crystal graph associated to the basic representation $V\left(\Lambda_{0}\right)$ of $\mathfrak{g}$ can now be realized as the set of all $p$-regular partitions, with a directed edge $\lambda \stackrel{i}{\longrightarrow} \mu$ of color $i \in I$ if $\mu$ is obtained from $\lambda$ by adding an $i$-cogood node (equivalently, $\lambda$ is obtained from $\mu$ by removing an $i$-good node). An example showing part of the crystal graph for $p=2$ is listed below.

2.5. The modular branching graph. Now we explain the relationship between the crystal graph and representation theory. The next lemma was first proved in [20], and in a different way in [11].

Lemma 2.9. Let $D$ be an irreducible $F S_{n}$-module and $i \in I$. Then, the module $e_{i} D$ (resp. $\left.f_{i} D\right)$ is either zero, or else is a self-dual $F S_{n-1}$ - (resp. $\left.F S_{n+1^{-}}\right)$module with irreducible socle and head isomorphic to each other.

Introduce the crystal operators $\tilde{e}_{i}, \tilde{f}_{i}$ : for an irreducible $F S_{n}$-module $D$, let

$$
\tilde{e}_{i} D:=\operatorname{socle}\left(e_{i} D\right), \quad \tilde{f}_{i} D:=\operatorname{socle}\left(f_{i} D\right) .
$$

In view of Lemma $2.9, \tilde{e}_{i} D$ and $\tilde{f}_{i} D$ are either zero or irreducible. Now define the modular branching graph: the vertices are the isomorphism classes of irreducible $F S_{n}$-modules for all $n \geq 0$, and there is a directed edge $[D] \stackrel{i}{\longrightarrow}$ $[E]$ of color $i$ if $E \cong \tilde{f}_{i} D$ (equivalently by Frobenius reciprocity, $D \cong \tilde{e}_{i} E$ ). The fundamental result is the following: 
The basic crystal graph of type $A_{1}^{(1)}$

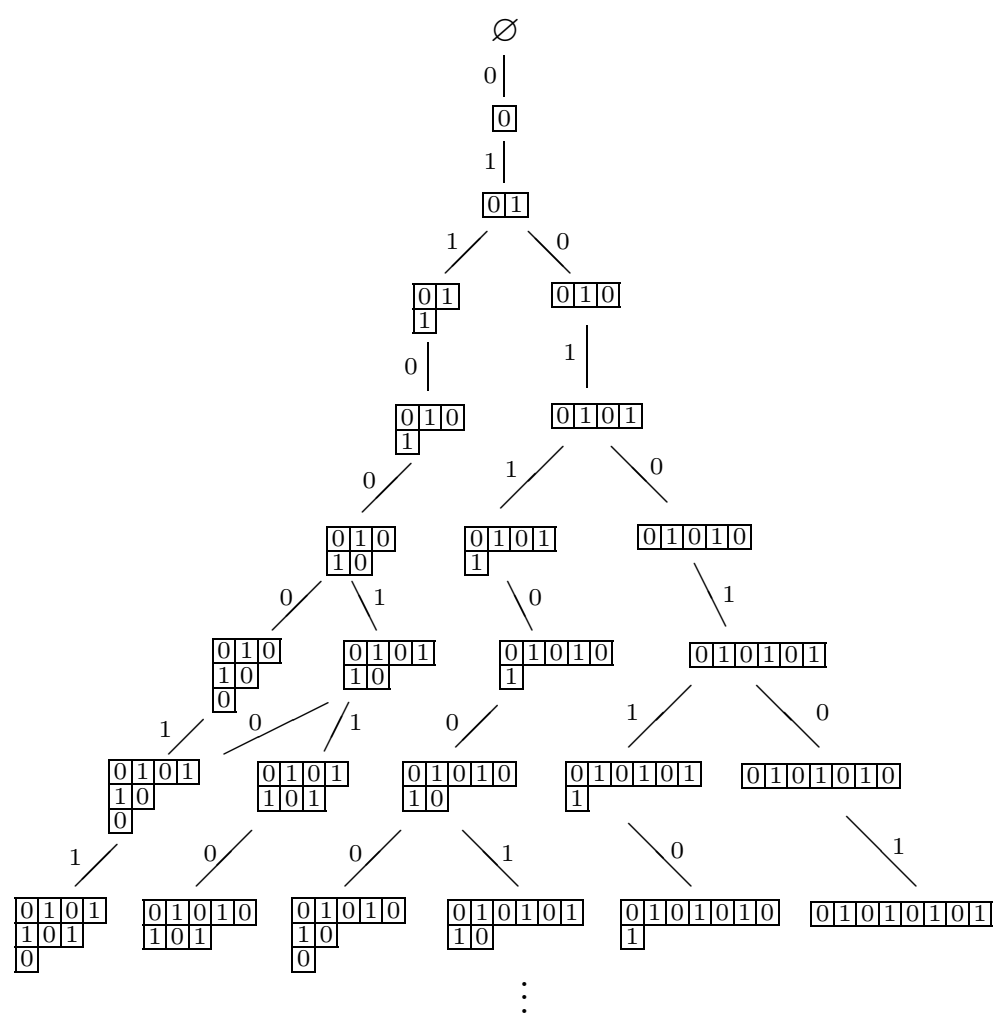

Theorem 2.10. The modular branching graph is uniquely isomorphic (as an I-colored, directed graph) to the crystal graph of §2.4.

This theorem was first stated in this way by Lascoux, Leclerc and Thibon [21]: they noticed that the combinatorics of Kashiwara's crystal graph as described by Misra and Miwa [26] is exactly the same as the modular branching graph first determined in [19]. A quite different and independent proof of Theorem 2.10 follows from the more general results of [9].

Theorem 2.10 has some important consequences. To start with, it implies that the isomorphism classes of irreducible $F S_{n}$-modules are parametrized by the vertices in the crystal graph, i.e. by $p$-regular partitions. For a $p$-regular partition $\lambda$ of $n$, we let $D^{\lambda}$ denote the corresponding irreducible $F S_{n}$-module. To be quite explicit about this labelling, choose a path

$$
\varnothing \stackrel{i_{1}}{\longrightarrow} \square \stackrel{i_{2}}{\longrightarrow} \cdots \stackrel{i_{n}}{\longrightarrow} \lambda
$$

in the crystal graph from the empty partition to $\lambda$, for $i_{1}, \ldots, i_{n} \in I$. Then,

$$
D^{\lambda}:=\tilde{f}_{i_{n}} \ldots \tilde{f}_{i_{1}} D^{\varnothing}
$$


where $D^{\varnothing}$ denotes the irreducible $F S_{0}$-module. Note the labelling of the irreducible module $D^{\lambda}$ defined here is known to agree with the standard labelling of James [13], although James' construction is quite different.

Let us state one more result about the structure of the modules $e_{i} D^{\lambda}$ and $f_{i} D^{\lambda}$, see $\left[2\right.$, Theorems $\left.\mathrm{E}, \mathrm{E}^{\prime}\right]$ for this and some other more detailed results.

Theorem 2.11. Let $\lambda$ be a p-regular partition of $n$.

(i) Suppose that $A$ is an $i$-removable node such that $\mu:=\lambda-\{A\}$ is $p$-regular. Then, $\left[e_{i} D^{\lambda}: D^{\mu}\right]$ is the number of $i$-normal nodes to the right of $A$ (counting $A$ itself), or 0 if $A$ is not $i$-normal.

(ii) Suppose that $B$ is an $i$-addable node such that $\nu:=\lambda \cup\{B\}$ is $p$ regular. Then, $\left[f_{i} D^{\lambda}: D^{\nu}\right]$ is the number of $i$-conormal nodes to the left of $B$ (counting $B$ itself), or 0 if $B$ is not $i$-conormal.

2.6. More on characters. Let $M$ be an $F S_{n}$-module. Define

$$
\varepsilon_{i}(M)=\max \left\{r \geq 0 \mid e_{i}^{r} M \neq 0\right\} \quad \varphi_{i}(M)=\max \left\{r \geq 0 \mid f_{i}^{r} M \neq 0\right\} .
$$

Note $\varepsilon_{i}(M)$ can be computed just from knowledge of the character of $M$ : it is the maximal $r$ such that $e^{\left(\ldots, i^{r}\right)}$ appears with non-zero coefficient in ch $M$. Less obviously, $\varphi_{i}(M)$ can also be read off from the character of $M$. By additivity of $f_{i}$, we may assume that $M$ belongs to the block $\gamma \in \Gamma_{n}$. Then

$$
\varphi_{i}(M)=\varepsilon_{i}(M)+\delta_{i, 0}-2 \gamma_{i}+\gamma_{i-1}+\gamma_{i+1},
$$

see $[9,12.6]$. We note the following extremely useful lemma from [11], see also $[9, \S 9]$ :

Lemma 2.12. Let $D$ be an irreducible F $S_{n}$-module, $\varepsilon=\varepsilon_{i}(D), \varphi=\varphi_{i}(D)$. Then, $e_{i}^{(\varepsilon)} D \cong \tilde{e}_{i}^{\varepsilon} D, f_{i}^{(\varphi)} D \cong \tilde{f}_{i}^{\varphi} D$.

The lemma implies that

$$
\varepsilon_{i}(D)=\max \left\{r \geq 0 \mid \tilde{e}_{i}^{r} D \neq 0\right\}, \quad \varphi_{i}(D)=\max \left\{r \geq 0 \mid \tilde{f}_{i}^{r} D \neq 0\right\} .
$$

Thus, $\varepsilon_{i}(D)$ can also be read off directly from the combinatorics: if $D \cong D^{\lambda}$, then $\varepsilon_{i}(D)$ is the number of '-'s in the reduced $i$-signature of $\lambda$. Similarly, $\varphi_{i}(D)$ is the number of ' + 's in the reduced $i$-signature of $\lambda$.

Now we can describe an inductive algorithm to determine the label of an irreducible $F S_{n}$-module $D$ purely from knowledge of its character ch $D$. Pick $i \in I$ such that $\varepsilon:=\varepsilon_{i}(D)$ is non-zero. Let $E=e_{i}^{(\varepsilon)} D$, an irreducible $F S_{n-\varepsilon^{-}}$ module with explicitly known character thanks to Lemmas 2.12, 2.6 and (6). By induction, the label of $E$ can be computed purely from knowledge of its character, say $E \cong D^{\lambda}$. Then, $D \cong \tilde{f}_{i}^{\varepsilon} E \cong D^{\mu}$ where $\mu$ is obtained from $\lambda$ by adding the rightmost $\varepsilon$ of the $i$-conormal nodes.

We would of course like to be able to reverse this process: given a $p$-regular partition $\lambda$ of $n$, we would like to be able to compute the character of the irreducible $F S_{n}$-module $D^{\lambda}$. One can compute a quite effective lower bound for this character inductively using the branching rules of Theorem 2.11. 
But only over $\mathbb{C}$ is this lower bound always correct: indeed if $p=0$ then $D^{\lambda}$ is equal to the Specht module $S^{\lambda}$ and

$$
\operatorname{ch} S^{\lambda}=\sum_{\left(i_{1}, \ldots, i_{n}\right)} e^{\left(i_{1}, \ldots, i_{n}\right)}
$$

summing over all paths $\varnothing \stackrel{i_{1}}{\longrightarrow} \square \stackrel{i_{2}}{\longrightarrow} \cdots \stackrel{i_{n}}{\longrightarrow} \lambda$ in the characteristic zero crystal graph (a.k.a. Young's partition lattice) from $\varnothing$ to $\lambda$. (Reducing the residues in (14) modulo $p$ in the obvious way gives the formal characters of the Specht module in characteristic $p$.) We refer to [32] for a concise self-contained approach to the complex representation theory of $S_{n}$ along the lines described here.

Now we explain how Lemma 2.12 can be used to describe some composition factors of Specht modules - this provides new non-trivial information on decomposition numbers which is difficult to obtain by other methods. The following result follows easily from Lemma 2.12 .

Lemma 2.13. Let $M$ be an $F S_{n}$-module and set $\varepsilon=\varepsilon_{i}(M)$. If $\left[e_{i}^{(\varepsilon)} M\right.$ : $\left.D^{\mu}\right]=m>0$ then $\tilde{f}_{i}^{\varepsilon} D^{\mu} \neq 0$ and $\left[M: \tilde{f}_{i}^{\varepsilon} D^{\mu}\right]=m$.

Example 2.14. Let $p=3$. By [13, Tables], the composition factors of the Specht module $S^{(6,4,2,1)}$ are $D^{(12,1)}, D^{(9,4)}, D^{\left(9,2^{2}\right)}, D^{(7,4,2)}, D^{(6,5,2)}$, $D^{(6,4,3)}$, and $D^{(6,4,2,1)}$, all appearing with multiplicity 1 . As $\varepsilon_{1}\left(S^{\left(6,4,2^{2}\right)}\right)=$ 1 (by (14) reduced modulo 3) and $e_{1} S^{\left(6,4,2^{2}\right)}=S^{(6,4,2,1)}$, application of Lemma 2.13 implies that the following composition factors appear in $S^{\left(6,4,2^{2}\right)}$ with multiplicity $1: D^{\left(12,1^{2}\right)}, D^{(9,4,1)}, D^{(9,3,2)}, D^{(8,4,2)}, D^{\left(6^{2}, 2\right)}, D^{\left(6,4^{2}\right)}$, and $D^{\left(6,4,2^{2}\right)}$.

Given $\underline{i}=\left(i_{1}, \ldots, i_{n}\right) \in I^{n}$ we can gather consecutive equal terms to write it in the form

$$
\underline{i}=\left(j_{1}^{m_{1}} \ldots j_{r}^{m_{r}}\right)
$$

where $j_{s} \neq j_{s+1}$ for all $1 \leq s<r$. For example $(2,2,2,1,1)=\left(2^{3} 1^{2}\right)$. Now, for an $F S_{n}$-module $M$, the tuple (15) is called extremal if

$$
m_{s}=\varepsilon_{j_{s}}\left(e_{j_{s+1}}^{m_{s+1}} \ldots e_{j_{r}}^{m_{r}} M\right)
$$

for all $s=r, r-1, \ldots, 1$. Informally speaking this means that among all the $n$-tuples $\underline{i}$ such that $M[\underline{i}] \neq 0$ we first choose those with the longest $j_{r}$-string in the end, then among these we choose the ones with the longest $j_{r-1}$-string preceding the $j_{r}$-string in the end, etc. By definition $M[\underline{i}] \neq 0$ if $\underline{i}$ is extremal for $M$.

Example 2.15. The formal character of the Specht module $S^{(5,2)}$ in characteristic 3 is

$$
\begin{aligned}
& e^{(0210201)}+2 e^{(0120201)}+2 e^{\left(02120^{2} 1\right)}+4 e^{\left(012^{2} 0^{2} 1\right)} \\
& +e^{(0212010)}+2 e^{\left(012^{2} 010\right)}+e^{(0120210)}+e^{(0120120)} .
\end{aligned}
$$

The extremal tuples are $\left(012^{2} 0^{2} 1\right),\left(012^{2} 010\right),(0120210)$, and (0120120). 
Our main result about extremal tuples is

Theorem 2.16. Let $\underline{i}=\left(i_{1}, \ldots, i_{n}\right)=\left(j_{1}^{m_{1}} \ldots j_{r}^{m_{r}}\right)$ be an extremal tuple for an irreducible $F S_{n}$-module $D^{\lambda}$. Then $D^{\lambda}=\tilde{f}_{i_{n}} \ldots \tilde{f}_{i_{1}} D^{\varnothing}$, and $\operatorname{dim} D^{\lambda}[\underline{i}]=$ $m_{1} ! \ldots m_{r}$ !. In particular, the tuple $\underline{i}$ is not extremal for any irreducible $D^{\mu} \nRightarrow D^{\lambda}$.

Proof. We apply induction on $r$. If $r=1$, then by considering possible $n$-tuples appearing in the Specht module $S^{\lambda}$, of which $D^{\lambda}$ is a quotient, we conclude that $n=1$ and $D=D^{(1)}$. So for $r=1$ the result is obvious. Let $r>1$. By definition of an extremal tuple, $m_{r}=\varepsilon_{j_{r}}\left(D^{\lambda}\right)$. So, in view of Lemmas 2.6 and 2.12, we have

$$
e_{j_{r}}^{m_{r}} D^{\lambda}=m_{r} ! \tilde{e}_{j_{r}}^{m_{r}} D^{\lambda}
$$

Moreover, $\left(j_{1}^{m_{1}} \ldots j_{r-1}^{m_{r-1}}\right)$ is clearly an extremal tuple for the irreducible module $\tilde{e}_{j_{r}}^{m_{r}} D^{\lambda}$. So the inductive step follows.

Corollary 2.17. If $M$ is an $F S_{n}$-module and $\underline{i}=\left(i_{1}, \ldots, i_{n}\right)=\left(j_{1}^{m_{1}} \ldots j_{r}^{m_{r}}\right)$ is an extremal tuple for $M$ then the multiplicity of $D^{\lambda}:=\tilde{f}_{i_{n}} \ldots \tilde{f}_{i_{1}} D^{\varnothing}$ as a composition factor of $M$ is $\operatorname{dim} M[i] /\left(m_{1} ! \ldots m_{r} !\right)$.

We note that for any tuple $\underline{i}$ represented in the form (15) and any $F S_{n^{-}}$ module $M$ we have that $\operatorname{dim} M[\underline{i}]$ is divisible by $m_{1} ! \ldots m_{r} !$. This follows from the properties of the principal series modules ('Kato modules') for degenerate affine Hecke algebras, see [11] for more details.

Example 2.18. In view of Corollary 2.17 extremal tuple $\left(012^{2} 0^{2} 1\right)$ in Example 2.15 yields the composition factor $D^{(5,2)}$ of $S^{(5,2)}$, while the extremal tuple (0120120) yields the composition factor $D^{(7)}$. It turns out that these are exactly the composition factors of $S^{(5,2)}$, see e.g. [13, Tables].

For more non-trivial examples let us consider a couple of Specht modules for $n=11$ in characteristic 3 . For $S^{\left(6,3,1^{2}\right)}$, Corollary 2.17 yields composition factors $D^{\left(6,3,1^{2}\right)}, D^{(7,3,1)}$, and $D^{(8,2,1)}$ but 'misses' $D^{(11)}$, and for $S^{\left(4,3,2^{2}\right)}$ we get hold of $D^{\left(4,3,2^{2}\right)}, D^{(5,3,2,1)}, D^{(8,2,1)}$, and $D^{(8,3)}$, but 'miss' $2 D^{(11)}$ and $D^{\left(5,4,1^{2}\right)}$, cf. [13, Tables].

We record here one other useful general fact about formal characters which follows from the Serre relations satisfied by the operators $e_{i}$ :

Lemma 2.19. Let $M$ be an $F S_{n}$-module. Assume $i, j, i_{1}, \ldots, i_{n-2} \in I$ and $i \neq j$.

(i) Assume that $|i-j|>1$. Then for any $1 \leq r \leq n-2$ we have

$$
\begin{aligned}
& \operatorname{dim} M\left[\left(i_{1}, \ldots, i_{r}, i, j, i_{r+1}, \ldots, i_{n-2}\right)\right] \\
= & \operatorname{dim} M\left[\left(i_{1}, \ldots, i_{r}, j, i, i_{r+1}, \ldots, i_{n-2}\right)\right] .
\end{aligned}
$$


(ii) Assume that $|i-j|=1$ and $p>2$. Then for any $1 \leq r \leq n-3$ we have

$$
\begin{aligned}
& 2 \operatorname{dim} M\left[\left(i_{1}, \ldots, i_{r}, i, j, i, i_{r+1}, \ldots, i_{n-3}\right)\right] \\
= & \operatorname{dim} M\left[\left(i_{1}, \ldots, i_{r}, i, i, j, i_{r+1}, \ldots, i_{n-3}\right)\right] \\
& +\operatorname{dim} M\left[\left(i_{1}, \ldots, i_{r}, j, i, i, i_{r+1}, \ldots, i_{n-3}\right)\right] .
\end{aligned}
$$

(iii) Assume that $|i-j|=1$ and $p=2$. Then for any $1 \leq r \leq n-4$ we have

$$
\begin{aligned}
& \operatorname{dim} M\left[\left(i_{1}, \ldots, i_{r}, i, i, i, j, i_{r+1}, \ldots, i_{n-4}\right)\right] \\
& +3 \operatorname{dim} M\left[\left(i_{1}, \ldots, i_{r}, i, j, i, i, i_{r+1}, \ldots, i_{n-4}\right)\right] \\
= & \operatorname{dim} M\left[\left(i_{1}, \ldots, i_{r}, j, i, i, i, i_{r+1}, \ldots, i_{n-4}\right)\right] \\
& +3 \operatorname{dim} M\left[\left(i_{1}, \ldots, i_{r}, i, i, j, i, i_{r+1}, \ldots, i_{n-4}\right)\right] .
\end{aligned}
$$

2.7. Blocks. Finally we discuss some properties of blocks, assuming now that $p \neq 0$. In view of Theorem 2.7(ii), the blocks of the $F S_{n}$ for all $n$ are in 1-1 correspondence with the non-zero weight spaces of the basic module $V\left(\Lambda_{0}\right)$ of $\mathfrak{g}=A_{p-1}^{(1)}$. So let us begin by describing these following [16, ch.12].

Let $P=\bigoplus_{i \in I} \mathbb{Z} \Lambda_{i} \oplus \mathbb{Z} \delta$ denote the weight lattice associated to $\mathfrak{g}$. Let $\alpha_{i}(i \in I)$ be the simple roots of $\mathfrak{g}$, defined from

$$
\alpha_{0}=2 \Lambda_{0}-\Lambda_{1}-\Lambda_{p-1}+\delta, \quad \alpha_{i}=2 \Lambda_{i}-\Lambda_{i+1}-\Lambda_{i-1} \quad(i \neq 0) .
$$

There is a positive definite symmetric bilinear form (.|.) on $\mathbb{R} \otimes_{\mathbb{Z}} P$ with respect to which $\alpha_{0}, \ldots, \alpha_{p-1}, \Lambda_{0}$ and $\Lambda_{0}, \ldots, \Lambda_{p-1}, \delta$ form a pair of dual bases. Let $W$ denote the Weyl group of $\mathfrak{g}$, the subgroup of $G L\left(\mathbb{R} \otimes_{\mathbb{Z}} P\right)$ generated by $s_{i}(i \in I)$, where $s_{i}$ is the reflection in the hyperplane orthogonal to $\alpha_{i}$. Then, by $[16,(12.6 .1)]$, the weight spaces of $V\left(\Lambda_{0}\right)$ are the weights

$$
\left\{w \Lambda_{0}-d \delta \mid w \in W, d \in \mathbb{Z}_{\geq 0}\right\}
$$

For a weight of the form $w \Lambda_{0}-d \delta$, we refer to $w \Lambda_{0}$ as the corresponding maximal weight, and $d$ as the corresponding depth.

There is a more combinatorial way of thinking of the weights. Following $[24, \mathrm{I} .1$, ex.8] and $[14, \S 2.7]$, to a $p$-regular partition $\lambda$ one associates the corresponding $p$-core $\tilde{\lambda}$ and $p$-weight $d$ : $\tilde{\lambda}$ is the partition obtained from $\lambda$ by successively removing as many hooks of length $p$ from the rim of $\lambda$ as possible, in such a way that at each step the diagram of a partition remains. The number of $p$-hooks removed is the $p$-weight $d$ of $\lambda$. The $p$-cores are in 1-1 correspondence with the maximal weights, i.e. the weights belonging to the $W$-orbit $W \Lambda_{0}$, and the $p$-weight corresponds to the notion of depth introduced in the previous paragraph, see $[21, \S 5.3]$ and $[22, \S 2]$ for the details.

Now Theorem 2.7(ii) gives yet another proof of the Nakayama conjecture: the $F S_{n}$-modules $D^{\lambda}$ and $D^{\mu}$ belong to the same block if and only if $\lambda$ and $\mu$ have the same $p$-core. We will also talk about the $p$-weight of a block $B$, namely, the $p$-weight of any $\lambda$ such that $D^{\lambda}$ belongs to $B$. 
The Weyl group $W$ acts on the $\mathfrak{g}$-module $R_{\mathbb{C}}$ from $\S 2.3$, the generator $s_{i}(i \in I)$ of $W$ acting by the familiar formula

$$
s_{i}=\exp \left(-e_{i}\right) \exp \left(f_{i}\right) \exp \left(-e_{i}\right)
$$

The resulting action preserves the Shapovalov form, and leaves the lattices $R$ and $R^{*}$ invariant. Moreover, $W$ permutes the weight spaces of $R_{\mathbb{C}}$ in the same way as its defining action on the weight lattice $P$. Since $W$ leaves $\delta$ invariant, it follows that the action is transitive on all weight spaces of the same depth. So using Theorem 2.7(iii) we see:

Theorem 2.20. Let $B$ and $B^{\prime}$ be blocks of symmetric groups with the same p-weight. Then, $B$ and $B^{\prime}$ are isometric, in the sense that there is an isomorphism between their Grothendieck groups that is an isometry with respect to the Cartan form.

The existence of such isometries was first noticed by Enguehard [8]. Implicit in Enguehard's paper is the following conjecture, made formally by Rickard: blocks $B$ and $B^{\prime}$ of symmetric groups with the same p-weight should be derived equivalent. This has been proved by Rickard for blocks of $p$-weight $\leq 5$. Moreover, it is now known by work of Marcus [25] and Chuang-Kessar [6] that the famous Abelian Defect Group Conjecture of Broué for symmetric groups follows from the Rickard's conjecture above.

There is one situation that is particularly straightforward, when there is actually a Morita equivalence between blocks of the same $p$-weight. This is a theorem of Scopes [34], though we are stating the result in a more Lie theoretic way following $[22, \S 8]$ :

Theorem 2.21. Let $\Lambda, \Lambda+\alpha_{i}, \ldots, \Lambda+r \alpha_{i}$ be an $\alpha_{i}$-string of weights of $V\left(\Lambda_{0}\right)$ (so $\Lambda-\alpha_{i}$ and $\Lambda+(r+1) \alpha_{i}$ are not weights of $V\left(\Lambda_{0}\right)$ ). Then the functors $f_{i}^{(r)}$ and $e_{i}^{(r)}$ define mutually inverse Morita equivalences between the blocks parametrized by $\Lambda$ and by $\Lambda+r \alpha_{i}$.

Proof. Since $e_{i}^{(r)}$ and $f_{i}^{(r)}$ are both left and right adjoint to one another, it suffices to check that $e_{i}^{(r)}$ and $f_{i}^{(r)}$ induce mutually inverse bijections between the isomorphism classes of irreducible modules belonging to the respective blocks. This follows by Lemma 2.12 .

Let us end the discussion with one new result here: we can in fact explicitly compute the determinant of the Cartan matrix of a block. The details of the proof will appear in [5]. Note in view of Theorem 2.20, the determinant of the Cartan matrix only depends on the $p$-weight of the block. Moreover, by Theorem 2.7(iii), we can work instead in terms of the Shapovalov form on $V\left(\Lambda_{0}\right)$. Using the explicit construction of the latter module over $\mathbb{Z}$ given in $[7]$, we show: 
Theorem 2.22. Let $B$ be a block of p-weight $d$ of $F S_{n}$. Then the determinant of the Cartan matrix of $B$ is $p^{N}$ where

$$
N=\sum_{\lambda=\left(1^{r_{1}} 2^{r_{2} \ldots} \ldots\right) \vdash d} \frac{r_{1}+r_{2}+\ldots}{p-1}\left(\begin{array}{c}
p-2+r_{1} \\
r_{1}
\end{array}\right)\left(\begin{array}{c}
p-2+r_{2} \\
r_{2}
\end{array}\right) \ldots
$$

\section{The Double Covers}

We turn now to the representation theory of the group algebra $F \widehat{S}_{n}$, where $\widehat{S}_{n}$ denotes one of the double covers of the symmetric group and $F$ is a field of characteristic $p \neq 2$. We will assume that $F$ contains square roots of (the images of) all integers, since that ensures that $F$ is a splitting field for $\widehat{S}_{n}$ for all $n$ (see [3, Remark 10.5]).

3.1. Analogues of the Jucys-Murphy elements. For definiteness, we work with the double cover $\widehat{S}_{n}$ defined by generators $\zeta, \hat{s}_{1}, \ldots, \hat{s}_{n-1}$ subject to the relations

$$
\zeta^{2}=1, \quad \zeta \hat{s}_{i}=\hat{s}_{i} \zeta, \quad \hat{s}_{i}^{2}=1, \quad \hat{s}_{i} \hat{s}_{j}=\zeta \hat{s}_{j} \hat{s}_{i}, \quad \hat{s}_{i} \hat{s}_{i+1} \hat{s}_{i}=\hat{s}_{i+1} \hat{s}_{i} \hat{s}_{i+1},
$$

for all admissible $i, j$ with $|i-j|>1$. Note right away that $1=\zeta_{+}+\zeta_{-}$ is a decomposition of the identity as a sum of mutually orthogonal central idempotents, where $\zeta_{ \pm}=(1 \mp \zeta) / 2$. So we can decompose

$$
F \widehat{S}_{n}=\zeta_{+} F \widehat{S}_{n} \oplus \zeta_{-} F \widehat{S}_{n}
$$

The algebra $\zeta_{+} F \widehat{S}_{n}$ is isomorphic to the group algebra $F S_{n}$ itself, so we focus our attention instead on the summand $S(n):=\zeta_{-} F \widehat{S}_{n}$.

The algebra $S(n)$ is the twisted group algebra of $S_{n}$ over $F$. It can be realized directly as the algebra generated by the elements $t_{i}:=\zeta_{-} \hat{s}_{i}$ subject only to the relations

$$
t_{i}^{2}=1, \quad t_{i} t_{j}=-t_{j} t_{i}, \quad t_{i} t_{i+1} t_{i}=t_{i+1} t_{i} t_{i+1},
$$

for admissible $i, j$ with $|i-j|>1$. For $1 \leq i<j \leq n$, let

$$
[i j]=-[j i]=(-1)^{j-i-1} t_{j-1} \ldots t_{i+1} t_{i} t_{i+1} \ldots t_{j-1} .
$$

These 'transpositions' satisfy the relations

$$
\begin{aligned}
& {[i j]^{2}=1, \quad[i j][k l]=-[k l][i j] \text { if }\{i, j\} \cap\{k, l\}=\varnothing,} \\
& {[i j][j k][i j]=[j k][i j][j k]=[k i] \text { for distinct } i, j, k}
\end{aligned}
$$

(cf. [36, (1.1)]). Finally, for distinct $1 \leq i_{1}, \ldots, i_{r} \leq n$, let

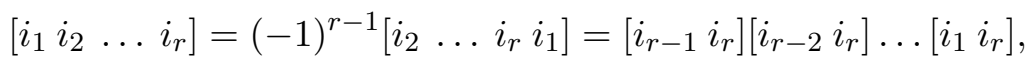

giving the ' $r$-cycles'.

For $1 \leq k \leq n$, the analogue of the Jucys-Murphy element is

$$
y_{k}:=\sum_{i=1}^{k-1}[i k]
$$


in particular, $y_{1}=0$. This definition appears in Sergeev [36]; Nazarov originally used a different approach [31]. One checks using the relations that:

$$
t_{i} y_{k}= \begin{cases}-y_{k} t_{i} & \text { if } i \neq k-1, k \\ -y_{k-1} t_{i}+1 & \text { if } i=k-1, \\ -y_{k+1} t_{i}+1 & \text { if } i=k .\end{cases}
$$

It follows that $y_{k} y_{l}=-y_{l} y_{k}$ if $k \neq l$. Now using these facts, it is easy to show:

(a) for $1 \leq k, l \leq n, y_{k}^{2}$ and $y_{l}^{2}$ commute;

(b) $t_{i}$ commutes with $y_{k}^{2}$ for $k \neq i, i+1$;

(c) $t_{i}$ commutes with $y_{i}^{2}+y_{i+1}^{2}$ and $y_{i}^{2} y_{i+1}^{2}$.

This implies:

Lemma 3.1. The symmetric polynomials in the elements $y_{1}^{2}, y_{2}^{2}, \ldots, y_{n}^{2}$ belong to the center of $S(n)$.

However it is not in general true that center of $S(n)$ equals the set of symmetric polynomials in the $y_{1}^{2}, \ldots, y_{n}^{2}$. We need to view $S(n)$ instead as a $\mathbb{Z}_{2}$-graded algebra - a superalgebra $S(n)=S(n)_{\overline{0}} \oplus S(n)_{\overline{1}}$ - by declaring that the generators $t_{i}$ are odd. Then:

Theorem 3.2. The even center of $S(n)$ (i.e. the space $Z(S(n))_{\overline{0}}$ of all central elements of degree $\overline{0})$ is the set of all symmetric polynomials in the $y_{1}^{2}, \ldots, y_{n}^{2}$.

Proof. $\quad$ For each $w \in S_{n}$, make a fixed choice for a representation of $w$ as a product of disjoint cycles (all of length $>1$ ). If $w=\left(i_{1} \ldots i_{a}\right)\left(j_{1} \ldots j_{b}\right) \ldots$ is this choice, define $[w]:=\left[i_{1} \ldots i_{a}\right]\left[j_{1} \ldots j_{b}\right] \cdots \in S(n)$. The $\left\{[w] \mid w \in S_{n}\right\}$ then form a basis for $S(n)$. We will say that $w \in S_{n}$ appears in $x \in S(n)$ if the coefficient of $[w]$ is non-zero when $x$ is expanded in terms of this basis.

Let $\lambda=\left(\lambda_{1} \geq \cdots \geq \lambda_{h}>0\right)$ be an odd partition of $n$, i.e. all its non-zero parts are odd. Define

$$
p_{\lambda}:=\sum_{w \in S_{n} / S_{\lambda}} y_{w 1}^{\lambda_{1}-1} y_{w 2}^{\lambda_{2}-1} \ldots y_{w h}^{\lambda_{h}-1} \in S(n),
$$

where $S_{\lambda}$ denotes the stabilizer of the $n$-tuple $\left(\lambda_{1}-1, \lambda_{2}-1, \ldots, \lambda_{h}-\right.$ $1,0, \ldots, 0)$ under the natural action of $S_{n}$ on $n$-tuples by place permutation. Also let

$$
\begin{aligned}
u_{\lambda}:=\left(n-\hat{\lambda}_{1}+1\right. & \ldots n)\left(n-\hat{\lambda}_{2}+1 \ldots n-\hat{\lambda}_{1}\right) \ldots \\
& \left(n-\hat{\lambda}_{h}+1 \ldots n-\hat{\lambda}_{h-1}\right) \in S_{n},
\end{aligned}
$$

where $\hat{\lambda}_{i}=\lambda_{1}+\cdots+\lambda_{i}$. Fix a total order $>$ on the odd partitions of $n$ so that $\lambda>\mu$ if either $\lambda$ has more non-zero parts than $\mu$, or if $\lambda, \mu$ have the same number of non-zero parts but $\lambda \triangleright \mu$ in the usual dominance ordering. By exactly the same argument as in the proof of $[29,1.9],\left[u_{\lambda}\right]$ appears in $p_{\lambda}$ but not in any $p_{\mu}$ with $\mu>\lambda$. This implies that the $p_{\lambda}$ are linearly independent, as $\lambda$ runs over all odd partitions of $n$. 
Finally, the $p_{\lambda}$ are symmetric polynomials in the $y_{1}^{2}, \ldots, y_{n}^{2}$ by definition. So we have shown that the dimension of the subspace of $S(n)$ spanned by the symmetric polynomials in the $y_{1}^{2}, \ldots, y_{n}^{2}$ is at least the number of odd partitions of $n$. On the other hand, by consideration of the conjugacy classes of even elements in $\widehat{S}_{n}$ (see [37, 2.1] or [33, p.172]), $\operatorname{dim} Z(S(n))_{\overline{0}}$ is equal to the number of odd partitions of $n$. So an application of Lemma 3.1 completes the proof.

3.2. Formal characters. Motivated in part by Theorem 3.2, we will be interested from now on in the $S(n)$-supermodules, i.e. the $\mathbb{Z}_{2}$-graded $S(n)$ modules where $S(n)$ is viewed as a $\mathbb{Z}_{2}$-graded algebra as before. We refer to $[3, \S 2]$ for basic notions here. Let us just recall here that there are two sorts of irreducible $S(n)$-supermodule $D$ : type M or type Q according to whether the endomorphism algebra $\operatorname{End}_{S(n)}(D)$ is one or two dimensional. In case $D$ has type M, it is irreducible when viewed as an ordinary $S(n)$-module. But if $D$ is of type $\mathrm{Q}$ it decomposes as $D=D_{+} \oplus D_{-}$where $D_{ \pm}$are non-isomorphic irreducible $S(n)$-submodules - but not subsupermodules - of $D$. Providing we keep track at all times of the type of an irreducible supermodule, we can easily recover results about ordinary representation theory. Incidentally, if $D$ has type $\mathrm{M}$ then $D \cong D \otimes \operatorname{sgn}$, and if $D$ has type $\mathrm{Q}$ then $D_{+} \cong D_{-} \otimes \mathrm{sgn}$.

Now we proceed along the lines of $\S 2.1$. Let $\ell=(p-1) / 2$ (resp. $\ell=\infty$ if $p=0)$. Let $I=\{0,1, \ldots, \ell\}$. Given a tuple $\underline{i}=\left(i_{1}, \ldots, i_{n}\right) \in I^{n}$ and an $S(n)$-supermodule $M$, we let

$$
M[\underline{i}]=\left\{v \in M \mid\left(y_{r}^{2}-\frac{i_{r}\left(i_{r}+1\right)}{2}\right)^{N} v=0 \text { for } N \gg 0 \text { and } r=1, \ldots, n\right\} .
$$

Lemma 3.3. Any $S(n)$-supermodule $M$ decomposes as $M=\bigoplus_{\underline{i} \in I^{n}} M[\underline{i}]$.

Proof. This follows from $[4,4.9,9.9]$ on noting that the image of our element $y_{k}^{2}$ under the map $\varphi: S(n) \rightarrow W(n)$ from $[4,9.8]$ is equal to one half of the image of the element denoted $x_{k}^{2}$ in [4].

We let $\Gamma_{n}$ denote the set of all $I$-tuples of non-negative integers summing to $n$, and define the weight of $\underline{i} \in I^{n}$ in the same way as in $\S 2.1$. Given $\gamma \in \Gamma_{n}$ and an $S(n)$-supermodule $M$, we set

$$
M[\gamma]:=\sum_{\underline{i} \in I^{n}} M[\underline{i}]
$$

as before. Theorem 3.2 and Lemma 3.3 imply:

Lemma 3.4. The decomposition $M=\bigoplus_{\gamma \in \Gamma_{n}} M[\gamma]$ is the precisely the decomposition of $M$ into superblocks as an $S(n)$-supermodule.

Now fix $\underline{i} \in I^{n}$ of weight $\gamma$. Consider the Clifford superalgebra with odd generators $c_{1}, \ldots, c_{n}$ subject to the relations

$$
c_{r} c_{s}=-c_{s} c_{r} \quad(r \neq s), \quad c_{r}^{2}=\frac{i_{r}\left(i_{r}+1\right)}{2} .
$$


By $[3,2.7,2.9,2.10]$, it has a unique irreducible supermodule $U(\underline{i})$, of type $\mathrm{M}$ if $\left(n-\gamma_{0}\right)$ is even, type $\mathbf{Q}$ if $\left(n-\gamma_{0}\right)$ is odd. Moreover,

$$
\operatorname{dim} U(\underline{i})=2^{\left\lfloor\frac{n-\gamma_{0}+1}{2}\right\rfloor} .
$$

Now suppose that $M$ is an $S(n)$-supermodule. The subspace $M[\underline{i}]$ is obviously invariant under the action of the subalgebra of $S(n)$ generated by the $y_{k}$. Moreover, these $y_{k}$ satisfy the above relations (20) on every irreducible constituent of $M[\underline{i}]$. This shows that $\operatorname{dim} M[\underline{i}]$ is divisible by $\operatorname{dim} U(\underline{i})$. Now define the formal character of $M$ by

$$
\operatorname{ch} M:=\sum_{\underline{i} \in I^{n}} \frac{\operatorname{dim} M[\underline{i}]}{\operatorname{dim} U(\underline{i})} e^{i},
$$

an element of the free $\mathbb{Z}$-module on basis $\left\{e^{\underline{i}} \mid \underline{i} \in I^{n}\right\}$. By $[4,5.12,9.10]$, we have:

Theorem 3.5. The characters of the pairwise inequivalent irreducible $S(n)$ supermodules are linearly independent. Moreover, the type of an irreducible $S(n)$-supermodule $D$ can be read off from its character: if $D$ belongs to the block $\gamma$ then $D$ is of type $\mathrm{M}$ if $\left(n-\gamma_{0}\right)$ is even, type $\mathrm{Q}$ if $\left(n-\gamma_{0}\right)$ is odd.

3.3. Induction and restriction operators. Next we introduce the analogues of the $i$-induction and $i$-restriction functors. Note $S(n-1)$ is naturally embedded in $S(n)$ as the subalgebra generated by $t_{1}, \ldots, t_{n-2}$. So we have natural restriction and induction functors $\operatorname{res}_{S(n-1)}^{S(n)}$ and $\operatorname{ind}_{S(n)}^{S(n+1)}:=$ $S(n+1) \otimes_{S(n)}$ ?.

Let $M$ be an $S(n)$-supermodule belonging to the block $\gamma \in \Gamma_{n}$. Given $i \in I$, define

$$
\begin{aligned}
\operatorname{res}_{i} M & \left.:=\left(\operatorname{res}_{S(n-1)}^{S(n)} M\right)[\gamma-i] \quad \text { (interpreted as zero in case } \gamma_{i}=0\right), \\
\operatorname{ind}_{i} M & :=\left(\operatorname{ind}_{S(n)}^{S(n+1)} M\right)[\gamma+i],
\end{aligned}
$$

where the notation $\gamma \pm i$ is as in $\S 2.2$. These definitions extend in an obvious way to give exact functors $\operatorname{res}_{i}$ and $\operatorname{ind}_{i}$. We note in particular that $\operatorname{res}_{i} M$ is the generalized eigenspace of eigenvalue $i(i+1) / 2$ for the action of $y_{n}^{2}$. By the definition and Lemma 3.3, we have:

Lemma 3.6. For an $S(n)$-supermodule $M$,

$$
\operatorname{res}_{S(n-1)}^{S(n)} M \cong \bigoplus_{i \in I} \operatorname{res}_{i} M, \quad \operatorname{ind}_{S(n)}^{S(n+1)} M \cong \bigoplus_{i \in I} \operatorname{ind}_{i} M .
$$

The next elementary lemma, proved rather indirectly in [4, 9.13,9.14], shows how the functors $\operatorname{res}_{i}$ and $\operatorname{ind}_{i}$ can be refined to obtain the correct definition of the operators $e_{i}$ and $f_{i}$ in this setting.

Lemma 3.7. Let $D$ be an irreducible $S(n)$-supermodule, and $i \in I$. 
(i) There is an $S(n-1)$-supermodule $e_{i} D$, unique up to isomorphism, such that

$$
\operatorname{res}_{i} D \cong \begin{cases}e_{i} D \oplus e_{i} D & \text { if } i \neq 0 \text { and } D \text { is of type } \mathbf{Q}, \\ e_{i} D & \text { if } i=0 \text { or } D \text { is of type } \mathrm{M} .\end{cases}
$$

(ii) There is an $S(n+1)$-supermodule $f_{i} D$, unique up to isomorphism, such that

$$
\operatorname{ind}_{i} D \cong \begin{cases}f_{i} D \oplus f_{i} D & \text { if } i \neq 0 \text { and } D \text { is of type } \mathbf{Q}, \\ f_{i} D & \text { if } i=0 \text { or } D \text { is of type } \mathbf{M} .\end{cases}
$$

We have now defined the operators $e_{i}, f_{i}(i \in I)$ on irreducible $S(n)$ supermodules (but note they are not functors defined on arbitrary supermodules, unlike before). Extending linearly, they induce operators also denoted $e_{i}, f_{i}$ at the level of characters. The effect of $e_{i}$ on characters is exactly the same as before:

$$
\text { if } \quad \operatorname{ch} M=\sum_{\underline{i} \in I^{n}} a_{\underline{i}} e^{\underline{i}} \quad \text { then } \quad \operatorname{ch}\left(e_{i} M\right)=\sum_{\underline{i} \in I^{n-1}} a_{\left(i_{1}, \ldots, i_{n-1}, i\right)} e^{\underline{i}} .
$$

This is one reason we have chosen to normalize characters the way we did in $(22)$.

There are also divided power operators $e_{i}^{(r)}$ and $f_{i}^{(r)}$. Again we just state a lemma characterizing them uniquely, rather than giving their explicit definition:

Lemma 3.8. Let $D$ be an irreducible $S(n)$-supermodule, and $i \in I$.

(i) There is an $S(n-r)$-supermodule $e_{i}^{(r)} D$, unique up to isomorphism, such that

$$
\left(\operatorname{res}_{i}\right)^{r} D \cong \begin{cases}\left(e_{i}^{(r)} D\right)^{\oplus r !} & \text { if } i=0, \\ \left(e_{i}^{(r)} D\right)^{\oplus 2^{\lfloor r / 2\rfloor} r !} & \text { if } i \neq 0 \text { and } D \text { is of type } \mathrm{M} \\ \left(e_{i}^{(r)} D\right)^{\oplus 2^{\lfloor(r+1) / 2\rfloor} r !} & \text { if } i \neq 0 \text { and } D \text { is of type } \mathbf{Q} .\end{cases}
$$

(ii) There is an $S(n+r)$-supermodule $f_{i}^{(r)} D$, unique up to isomorphism, such that

$$
\left(\operatorname{ind}_{i}\right)^{r} D \cong \begin{cases}\left(f_{i}^{(r)} D\right)^{\oplus r !} & \text { if } i=0, \\ \left(f_{i}^{(r)} D\right)^{\oplus 2^{\lfloor r / 2\rfloor} r !} & \text { if } i \neq 0 \text { and } D \text { is of type } \mathrm{M} \\ \left(f_{i}^{(r)} D\right)^{\oplus 2^{\lfloor(r+1) / 2\rfloor} r !} & \text { if } i \neq 0 \text { and } D \text { is of type } \mathrm{Q} .\end{cases}
$$

Note comparing Lemmas 3.6 and 3.8, we see that

$$
e_{i}^{r}=r ! e_{i}^{(r)}, \quad f_{i}^{r}=r ! f_{i}^{(r)}
$$

at the level of characters. 
3.4. The affine Kac-Moody algebra. Now things go in almost exactly the same way as $\S 2.3$. Let $R_{n}$ denote the character ring of $S(n)$, i.e. the free $\mathbb{Z}$-module spanned by the formal characters of the irreducible $F S_{n^{-}}$ supermodules, and let $R_{n}^{*}$ denote the $\mathbb{Z}$-submodule of $R_{n}$ spanned by the formal characters of the projective indecomposable $S(n)$-supermodules. Let

$$
R=\bigoplus_{n \geq 0} R_{n}, \quad R^{*}=\bigoplus_{n \geq 0} R_{n}^{*} \subseteq R .
$$

The $e_{i}$ and $f_{i}$ induce $\mathbb{Z}$-linear operators on $R$, stabilizing $R^{*}$. Extending scalars we get $\mathbb{C}$-linear operators $e_{i}$ and $f_{i}$ on $R_{\mathbb{C}}:=\mathbb{C} \otimes_{\mathbb{Z}} R=\mathbb{C} \otimes_{\mathbb{Z}}$ $R^{*}$. Finally, we have the symmetric Cartan form on $R_{\mathbb{C}}$, with respect to which the characters of the projective indecomposable supermodules and the irreducible supermodules form a pair of dual bases.

Theorem 3.9. The operators $e_{i}$ and $f_{i}(i \in I)$ on $R_{\mathbb{C}}$ satisfy the defining relations of the Chevalley generators of the affine Kac-Moody Lie algebra $\mathfrak{g}$ of type $A_{p-1}^{(2)}$ (resp. $B_{\infty}$ in case $\left.p=0\right)$, see [16]. Moreover, viewing $R_{\mathbb{C}}$ as a $\mathfrak{g}$-module in this way,

(i) $R_{\mathbb{C}}$ is isomorphic to the basic representation $V\left(\Lambda_{0}\right)$ of $\mathfrak{g}$, generated by the highest weight vector $e^{0}$ (the character of the irreducible FS(0)module);

(ii) the decomposition of $R_{\mathbb{C}}$ into superblocks coincides with its weight space decomposition with respect to the standard Cartan subalgebra of $\mathfrak{g}$;

(iii) the Cartan form on $R_{\mathbb{C}}$ coincides with the Shapovalov form satisfying $\left(e^{0}, e^{0}\right)=1$

(iv) the lattice $R^{*} \subset R_{\mathbb{C}}$ is the $\mathbb{Z}$-submodule of $R_{\mathbb{C}}$ generated by $e^{0}$ under the action of the operators $f_{i}^{(r)}=f_{i}^{r} / r !(i \in I, r \geq 0)$;

(v) the lattice $R \subset R_{\mathbb{C}}$ is the dual lattice to $R^{*}$ under the Shapovalov form.

This was proved in $[4,7.16]$.

3.5. The crystal graph. Next we describe the crystal underlying the basic representation $V\left(\Lambda_{0}\right)$ of the affine Kac-Moody algebra $\mathfrak{g}=A_{p-1}^{(2)}$. This explicit combinatorics is due to Kang [17]. We will work now with the set of all $p$-strict partitions, i.e. the partitions $\lambda=\left(\lambda_{1}, \lambda_{2}, \ldots\right)$ with the property that $p$ divides $\lambda_{r}$ whenever $\lambda_{r}=\lambda_{r+1}$. For example, the 0 -strict partitions are the partitions with no repeated non-zero parts.

Given a $p$-strict partition $\lambda$, we label its nodes with residues taken from the set $I=\{0,1, \ldots, \ell\}$ (recall $\ell=(p-1) / 2$ or $\infty$ if $p=0)$. The labelling depends only on the column and follows the repeating pattern

$$
0,1, \ldots, \ell-1, \ell, \ell-1, \ldots, 1,0
$$

starting fom the first column and going to the right, see Example 3.10 below. The residue of the node $A$ is denoted res $A$. 
Let $i \in I$ be some fixed residue. A node $A=(r, s) \in \lambda$ is called $i$ removable (for $\lambda$ ) if one of the following holds:

(R1) res $A=i$ and $\lambda-\{A\}$ is again a $p$-strict partition;

(R2) the node $B=(r, s+1)$ immediately to the right of $A$ belongs to $\lambda$, res $A=\operatorname{res} B=i$, and both $\lambda-\{B\}$ and $\lambda-\{A, B\}$ are $p$-strict partitions.

Similarly, a node $B=(r, s) \notin \lambda$ is called $i$-addable (for $\lambda$ ) if one of the following holds:

(A1) res $B=i$ and $\lambda \cup\{B\}$ is again an $p$-strict partition;

(A2) the node $A=(r, s-1)$ immediately to the left of $B$ does not belong to $\lambda$, res $A=\operatorname{res} B=i$, and both $\lambda \cup\{A\}$ and $\lambda \cup\{A, B\}$ are $p$-strict partitions.

We note that (R2) and (A2) above are only possible in case $i=0$.

Now label all $i$-addable nodes of the diagram $\lambda$ by + and all $i$-removable nodes by - . The $i$-signature of $\lambda$ is the sequence of pluses and minuses obtained by going along the rim of the Young diagram from bottom left to top right and reading off all the signs. The reduced $i$-signature of $\lambda$ is obtained from the $i$-signature by successively erasing all neighbouring pairs of the form +-. Warning: for historical reasons, the rule for obtaining the reduced $i$-signature here is different from in $\S 2.4$ : there one deleted pairs of the form -+ .

Note the reduced $i$-signature always looks like a sequence of -'s followed by + 's. Nodes corresponding to a - in the reduced $i$-signature are called $i$-normal, nodes corresponding to a + are called $i$-conormal. The rightmost $i$-normal node (corresponding to the rightmost - in the reduced $i$-signature) is called $i$-good, and the leftmost $i$-conormal node (corresponding to the leftmost + in the reduced $i$-signature) is called $i$-cogood.

Example 3.10. Let $p=5$, so $\ell=2$. The partition $\lambda=(16,11,10,10,9,5,1)$ is $p$-strict, and its residues are as follows:

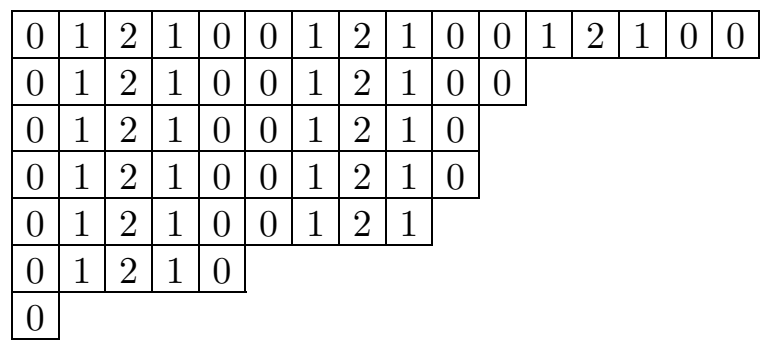


The 0-addable and 0-removable nodes are as labelled in the diagram:

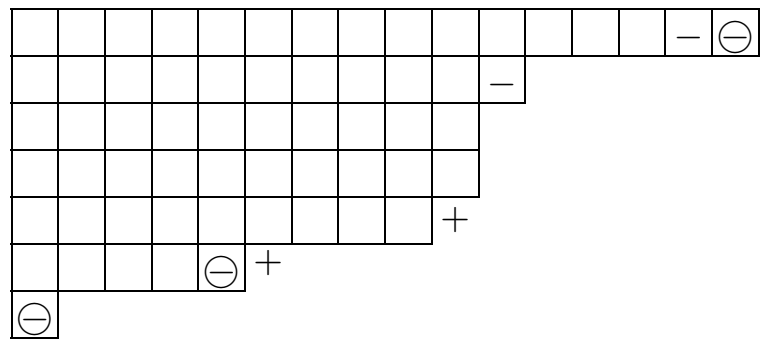

Hence, the 0 -signature of $\lambda$ is,,,,,,--++--- and the reduced 0 -signature is,,--- . Note the nodes corresponding to the -'s in the reduced $0-$ signature have been circled in the above diagram. So, there are three 0normal nodes, the rightmost of which is 0 -good; there are no 0 -conormal or 0 -cogood nodes.

Finally we call a $p$-strict partition $\lambda$ restricted if either $p=0$ or

$$
\begin{array}{ll}
\lambda_{i}-\lambda_{i+1}<p & \text { if } p \mid \lambda_{i}, \\
\lambda_{i}-\lambda_{i+1} \leq p & \text { if } p \nmid \lambda_{i},
\end{array}
$$

for each $i=1,2, \ldots$. The crystal graph associated to the basic representation $V\left(\Lambda_{0}\right)$ of $\mathfrak{g}$ is now the set of all restricted $p$-strict partitions of $n$, for all $n \geq 0$, with a directed edge $\lambda \stackrel{i}{\longrightarrow} \mu$ of color $i \in I$ if $\mu$ is obtained from $\lambda$ by adding an $i$-cogood node (equivalently, $\lambda$ is obtained from $\mu$ by removing an $i$-good node). For an example in case $p=3$, see below.

3.6. The modular branching graph. The connection between the crystal graph and the representation theory of $S(n)$ now proceeds in exactly the same way as in $\S 2.5$. The starting point is the following lemma proved in $[4,6.6,9.13,9.14]$ :

Lemma 3.11. Let $D$ be an irreducible $S(n)$-supermodule and $i \in I$. Then, the supermodule $e_{i} D$ (resp. $\left.f_{i} D\right)$ is either zero, or else is a self-dual $S(n-1)$ (resp. $S(n+1)$-) supermodule with irreducible socle and head isomorphic to each other.

The crystal operators $\tilde{e}_{i}, \tilde{f}_{i}$ are defined on an irreducible $S(n)$-supermodule $D$ by

$$
\tilde{e}_{i} D:=\operatorname{socle}\left(e_{i} D\right), \quad \tilde{f}_{i} D:=\operatorname{socle}\left(f_{i} D\right) .
$$

In view of Lemma $3.11, \tilde{e}_{i} D$ and $\tilde{f}_{i} D$ are either zero or irreducible. The modular branching graph has vertices equal to the isomorphism classes of irreducible $S(n)$-supermodules for all $n \geq 0$, and there is a directed edge $[D] \stackrel{i}{\longrightarrow}[E]$ of color $i$ if $E \cong \tilde{f}_{i} D$ (equivalently by Frobenius reciprocity, $D \cong \tilde{e}_{i} E$ ). The fundamental result is the following:

Theorem 3.12. The modular branching graph is uniquely isomorphic (as an I-colored, directed graph) to the crystal graph of $\S 3.5$. 
The basic crystal graph of type $A_{2}^{(2)}$

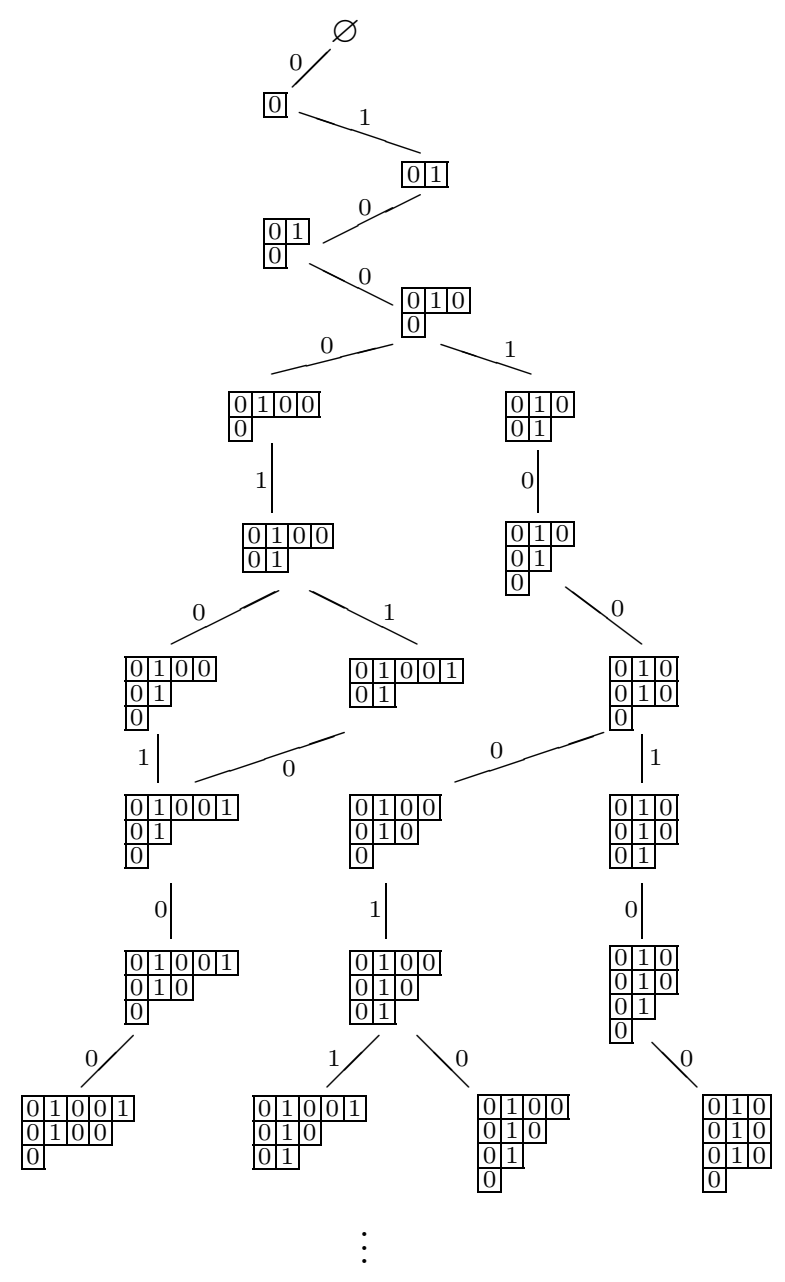

As before, Theorem 3.12 yields a parametrization of the isomorphism classes of irreducible $S(n)$-supermodules by the vertices of the crystal graph. Precisely, if $\lambda$ is a restricted $p$-strict partition of $n$, choose a path

$$
\varnothing \stackrel{i_{1}}{\longrightarrow} \square \stackrel{i_{2}}{\longrightarrow} \cdots \stackrel{i_{n}}{\longrightarrow} \lambda
$$

in the crystal graph from the empty partition to $\lambda$, for $i_{1}, \ldots, i_{n} \in I$. Define

$$
D(\lambda):=\tilde{f}_{i_{n}} \ldots \tilde{f}_{i_{1}} D(\varnothing),
$$

where $D(\varnothing)$ denotes the irreducible $S(0)$-supermodule. Using Theorem 3.5 for the second statement, we have:

Corollary 3.13. The supermodules

$$
\{D(\lambda) \mid \lambda \text { a restricted } p \text {-strict partition of } n\}
$$


form a complete set of inequivalent irreducible $S(n)$-supermodules. Moreover, letting $h_{p^{\prime}}(\lambda)$ denote the number of parts of $\lambda$ not divisible by $p, D(\lambda)$ has type $\mathrm{M}$ or $\mathrm{Q}$ according to whether $\left(n-h_{p^{\prime}}(\lambda)\right)$ is even or odd respectively.

This corollary solves the labelling problem for irreducible representations of the double covers of the symmetric group. That the restricted $p$-strict partitions should be suitable for this was suggested first by Leclerc and Thibon [23]. Note we gave an entirely different construction of the irreducible $S(n)$-supermodules, also labelled by restricted $p$-strict partitions, in [3]. At present we cannot prove that the two labellings agree, though we expect this to be the case. Another problem, which would be very useful in applications, is to find a representation theoretic interpretation of the normal and conormal nodes along the lines of Theorem 2.11.

It is easy to obtain a parametrization of the irreducible $S(n)$-modules (not super) from Corollary 3.13. If $D(\lambda)$ has type M, it is an irreducible $S(n)$ module in the ordinary sense, but we denote it by $D(\lambda, 0)$ to make it clear we have forgotten the $\mathbb{Z}_{2}$-grading. But if $D(\lambda)$ has type $\mathbf{Q}$, it decomposes as

$$
D(\lambda)=D(\lambda,+) \oplus D(\lambda,-)
$$

as an $S(n)$-module. Then a complete set of pairwise non-isomorphic $S(n)$ modules is given by the $\{D(\lambda, 0)\} \cup\{D(\mu, \pm)\}$ as $\lambda$ runs over all restricted $p$-strict partitions of $n$ with $n-h_{p^{\prime}}(\lambda)$ even and as $\mu$ runs over all restricted $p$-strict partitions of $n$ with $n-h_{p^{\prime}}(\mu)$ odd.

Let us finally note that there are analogues of the results of $\S 2.6$ too. For an irreducible $S(n)$-supermodule $D$, set

$$
\begin{aligned}
\varepsilon_{i}(D) & =\max \left\{r \geq 0 \mid\left(\operatorname{res}_{i}\right)^{r} D \neq 0\right\} \\
\varphi_{i}(D) & =\max \left\{r \geq 0 \mid\left(\operatorname{ind}_{i}\right)^{r} D \neq 0\right\} .
\end{aligned}
$$

As before $\varepsilon_{i}(D)$ can be computed just from knowledge of the character of $D$. Moreover, for $D$ belonging to the block $\gamma \in \Gamma_{n}, \varphi_{i}(D)$ is related to $\varepsilon_{i}(D)$ by the formula

$$
\varphi_{i}(D)= \begin{cases}\varepsilon_{i}(D)+1-2 \gamma_{0}+2 \gamma_{1} & i=0 \\ \varepsilon_{i}(D)-2 \gamma_{i}+\gamma_{i-1}+\gamma_{i+1} & i=1, \ldots, \ell-2, \\ \varepsilon_{i}(D)-2 \gamma_{\ell-1}+\gamma_{\ell-2}+2 \gamma_{\ell} & i=\ell-1 \\ \varepsilon_{i}(D)-2 \gamma_{\ell}+\gamma_{\ell-1} & i=\ell\end{cases}
$$

for $\ell \neq 1$, or

$$
\varphi_{0}(D)=\varepsilon_{0}(D)+1-2 \gamma_{0}+4 \gamma_{1}, \quad \varphi_{1}(D)=\varepsilon_{1}(D)-2 \gamma_{1}+\gamma_{0} .
$$

if $\ell=1$.

The analogue of Lemma 2.12 holds exactly as stated before, so $\varepsilon_{i}(D(\lambda))$ and $\varphi_{i}(D(\lambda))$ can also be read off directly from the crystal graph as the number of '-'s resp. ' + 's in the reduced $i$-signature of $\lambda$. So one obtains an inductive algorithm to determine the label of an irreducible $S(n)$-supermodule $D$ purely from knowledge of its character ch $D$, in exactly the same way as before. 
3.7. Blocks. To state some results about blocks, let $\mathfrak{g}=A_{p-1}^{(2)}$ and let $P=\bigoplus_{i \in I} \mathbb{Z} \Lambda_{i} \oplus \mathbb{Z} \delta$ be the associated weight lattice. The simple roots $\alpha_{i}(i \in I)$ can be defined by

$$
\begin{aligned}
\alpha_{0} & =2 \Lambda_{0}-\Lambda_{1}, \\
\alpha_{1} & =-2 \Lambda_{0}+2 \Lambda_{1}-\Lambda_{2}, \quad(i=2, \ldots, \ell-1), \\
\alpha_{i} & =-\Lambda_{i-1}+2 \Lambda_{i}-\Lambda_{i+1} \quad \\
\alpha_{\ell} & =-2 \Lambda_{\ell-1}+2 \Lambda_{\ell}+\delta
\end{aligned}
$$

if $\ell \neq 1$, and

$$
\alpha_{0}=2 \Lambda_{0}-\Lambda_{1}, \quad \alpha_{1}=-4 \Lambda_{0}+2 \Lambda_{1}+\delta
$$

if $\ell=1$. Let (.|.) be the positive definite symmetric bilinear form on $\mathbb{R} \otimes_{\mathbb{Z}} P$ with respect to which $2 \alpha_{0}, \alpha_{1}, \ldots, \alpha_{\ell-1}, \frac{1}{2} \alpha_{\ell}, \Lambda_{0}$ and $\Lambda_{0}, \ldots, \Lambda_{\ell}, \delta$ form a pair of dual bases. The Weyl group $W$ is the subgroup of $G L\left(\mathbb{R} \otimes_{\mathbb{Z}} P\right)$ generated by the reflections $s_{i}(i \in I)$ in the hyperplanes orthogonal to the $\alpha_{i}$. Then, by $[16,(12.6 .1)]$, the weights of the $\mathfrak{g}$-module $V\left(\Lambda_{0}\right)$ are the

$$
\left\{w \Lambda_{0}-d \delta \mid w \in W, d \in \mathbb{Z}_{\geq 0}\right\} .
$$

There are combinatorial notions paralleling this description of weights, namely, Morris' notions of $p$-bar core and $p$-bar weight [27]. Let $\lambda$ be a $p$-strict partition. By a $p$-bar of $\lambda$, we mean one of the following:

(B1) the rightmost $p$ nodes of row $i$ of $\lambda$ if $\lambda_{i} \geq p$ and either $p \mid \lambda_{i}$ or $\lambda$ has no row of length $\left(\lambda_{i}-p\right)$;

(B2) the set of nodes in rows $i$ and $j$ of $\lambda$ if $\lambda_{i}+\lambda_{j}=p$.

If $\lambda$ has no $p$-bars, it is called a $p$-bar core. In general, the $p$-bar core $\tilde{\lambda}$ of $\lambda$ is obtained by successively removing $p$-bars, reordering the rows each time so that the result is still a $p$-strict partition, until it is reduced to a core. The $p$-bar weight $d$ of $\lambda$ is then the total number of $p$-bars that get removed.

Now we get the classification of superblocks from Theorem 3.9(ii): irreducible $S(n)$-supermodules $D(\lambda)$ and $D(\mu)$ belong to the same superblock if and only if $\lambda$ and $\mu$ have the same $p$-bar core. Moreover, exactly as for Theorem 2.20, superblocks $B$ and $B^{\prime}$ of the same $p$-bar weight are isometric, in the sense that there is an isomorphism between their Grothendieck groups (induced by the action of $W$ ) which is an isometry with respect to the Cartan pairing.

It is more natural from the point of view of finite group theory to ask for a description of the ordinary (not super) blocks of $S(n)$. This does not seem to follow easily from the present theory, unless we invoke the work of Humphreys [12] (in fact, all we need from [12] is to know the number of ordinary blocks). There are two sorts of superblocks, of type $\mathrm{M}$ or $\mathrm{Q}$ according to whether all the irreducible supermodules belonging to the superblock are of type $\mathbf{M}$ or $\mathbf{Q}$. Corresponding to superblocks of type $\mathrm{M}$, there are ordinary blocks all of whose irreducible modules are of the form $D(\lambda, 0)$, and $D(\lambda, 0)$ 
and $D(\mu, 0)$ belong to the same block if and only if $\lambda$ and $\mu$ have the same $p$ bar core. Corresponding to superblocks of type $\mathrm{Q}$, there are ordinary blocks consisting of irreducible modules of the form $D(\lambda, \pm)$. Again, $D(\lambda, \varepsilon)$ and $D(\mu, \delta)$ belong to the same block if and only if $\lambda$ and $\mu$ have the same $p$-bar core, with one exception: if $\lambda$ is itself a $p$-bar core, then $D(\lambda,+)$ and $D(\lambda,-)$ are in different blocks.

Finally let us state the analogue of Theorem 2.22 giving the Cartan determinant of a superblock, see [5]:

Theorem 3.14. Let $B$ be a superblock of p-bar weight d of $S(n)$. Then the determinant of the Cartan matrix of $B$ is $p^{N}$ where $N$ equals

$$
\sum \frac{2 r_{1}+2 r_{3}+2 r_{5}+\ldots}{p-1}\left(\begin{array}{c}
\frac{p-3}{2}+r_{1} \\
r_{1}
\end{array}\right)\left(\begin{array}{c}
\frac{p-3}{2}+r_{2} \\
r_{2}
\end{array}\right)\left(\begin{array}{c}
\frac{p-3}{2}+r_{3} \\
r_{3}
\end{array}\right) \ldots,
$$

the sum being over all partitions $\lambda=\left(1^{r_{1}} 2^{r_{2}} \ldots\right) \vdash d$.

Note for superblocks of type $M$, this same formula gives the Cartan determinant of the corresponding ordinary block of $S(n)$. It appears that the same is true for superblocks of type $Q$, but we do not see how to deduce this from the theorem: the problem is that the ordinary block has twice as many irreducibles as in the corresponding superblock.

\section{REFERENCES}

[1] S. Ariki, On the decomposition numbers of the Hecke algebra of type $G(m, 1, n), J$. Math. Kyoto Univ. 36 (1996), 789-808.

[2] J. Brundan and A. Kleshchev, Translation functors for general linear and symmetric groups, Proc. London Math. Soc. 80 (2000), 75-106.

[3] J. Brundan and A. Kleshchev, Projective representations of the symmetric group via Sergeev duality, to appear in Math. Z..

[4] J. Brundan and A. Kleshchev, Hecke-Clifford superalgebras, crystals of type $A_{2 \ell}^{(2)}$ and modular branching rules for $\widehat{S}_{n}$, Represent. Theory 5 (2001), 317-403.

[5] J. Brundan and A. Kleshchev, Cartan determinants and Shapovalov forms, preprint, University of Oregon, 2001

[6] J. Chuang and R. Kessar, Symmetric groups, wreath products, Morita equivalences, and Broué's abelian defect group conjecture, preprint, 2001.

[7] C. De Concini, V. Kac and D. Kazhdan, "Boson-Fermion correspondence over $\mathbb{Z} "$, in: Infinite-dimensional Lie algebras and groups (Luminy-Marseille, 1988), Adv. Ser. Math. Phys. 7 (1989), 124-137.

[8] M. Enguehard, Isométries parfaites entre blocs de groupes symétriques, Astérisque 181-182 (1990), 157-171.

[9] I. Grojnowski, Affine $\widehat{\mathfrak{s l}}_{p}$ controls the modular representation theory of the symmetric group and related Hecke algebras, preprint, 1999.

[10] I. Grojnowski, Blocks of the cyclotomic Hecke algebra, preprint, 1999.

[11] I. Grojnowski and M. Vazirani, Strong multiplicity one theorem for affine Hecke algebras of type A, Transf. Groups. 6 (2001), 143-155.

[12] J. F. Humphreys, Blocks of projective representations of the symmetric groups, J. London Math. Soc. 33 (1986), 441-452.

[13] G. D. James, The representation theory of the symmetric groups, Lecture Notes in Math., vol. 682, Springer-Verlag, 1978.

[14] G. James and A. Kerber, The Representation Theory of the Symmetric Groups. Addison-Wesley, London, 1980. 
[15] A. Jucys, Symmetric polynomials and the center of the symmetric group ring, Report Math. Phys. 5 (1974), 107-112.

[16] V. Kac, Infinite dimensional Lie algebras, Cambridge University Press, third edition, 1995.

[17] S.-J. Kang, Crystal bases for quantum affine algebras and combinatorics of Young walls, preprint, Seoul National University, 2000.

[18] M. Kashiwara, "On crystal bases", in: Representations of groups (Banff 1994), CMS Conf. Proc. 16 (1995), 155-197.

[19] A. Kleshchev, Branching rules for modular representations of symmetric groups II, J. reine angew. Math. 459 (1995), 163-212.

[20] A. Kleshchev. Branching rules for modular representations of symmetric groups. III. Some corollaries and a problem of Mullineux. J. London Math. Soc. (2) 54 (1996), $25-38$.

[21] A. Lascoux, B. Leclerc and J.-Y. Thibon, Hecke algebras at roots of unity and crystal bases of quantum affine algebras, Comm. Math. Phys. 181 (1996), 205-263.

[22] B. Leclerc and H. Miyachi, Some closed formulas for canonical bases of Fock spaces, preprint, 2001.

[23] B. Leclerc and J.-Y. Thibon, $q$-Deformed Fock spaces and modular representations of spin symmetric groups, J. Phys. A 30 (1997), 6163-6176.

[24] I. G. Macdonald, Symmetric functions and Hall polynomials, Oxford Mathematical Monographs, second edition, OUP, 1995.

[25] A. Marcus, On equivalences between blocks of group algebras: reduction to the simple components, J. Algebra, 184 (1996), 372-396.

[26] K. Misra and T. Miwa, Crystal base for the basic representation of $U_{q}(\mathfrak{s l}(n))$, Comm. Math. Phys. 134 (1990), 79-88.

[27] A.O. Morris, The spin representations of the symmetric group, Canad. J. Math. 17 (1965), 543-549.

[28] G. Murphy, A new construction of Young's seminormal representations of the symmetric groups, J. Algebra 69 (1981), 287-297.

[29] G. Murphy, The idempotents of the symmetric group and Nakayama's conjecture, $J$. Algebra 81 (1983), 258-265.

[30] M. Nazarov, Young's orthogonal form of irreducible projective representations of the symmetric group, J. London Math. Soc. (2) 42 (1990), 437-451.

[31] M. Nazarov, Young's symmetrizers for projective representations of the symmetric group, Advances Math. 127 (1997), 190-257.

[32] A. Okounkov and A. Vershik, A new approach to representation theory of symmetric groups. Selecta Math. (N.S.) 2 (1996), 581-605.

[33] I. Schur, Über die Darstellung der symmetrischen und der alternierenden Gruppe durch gebrochene lineare Substitutionen, J. Reine Angew. Math. 139 (1911), 155250.

[34] J. Scopes, Cartan matrices and Morita equivalence for blocks of the symmetric groups. J. Algebra 142 (1991), 441-455.

[35] A. N. Sergeev, Tensor algebra of the identity representation as a module over the Lie superalgebras $G L(n, m)$ and $Q(n)$, Math. USSR Sbornik 51 (1985), 419-427.

[36] A. N. Sergeev, The Howe duality and the projective representations of symmetric groups, Represent. Theory 3 (1999), 416-434.

[37] J. Stembridge, Shifted tableaux and the projective representations of symmetric groups, Advances in Math. $\mathbf{7 4}$ (1989), 87-134.

[38] M. Vazirani, Irreducible modules over the affine Hecke algebra: a strong multiplicity one result, Ph.D. thesis, UC Berkeley, 1999.

Department of Mathematics, University of Oregon, Eugene, OR 97403, USA

E-mail address: brundan@darkwing.uoregon.edu, klesh@math.uoregon.edu 